\title{
Hardness Behavior of Alumina-Zirconia Nanocomposites Synthesized by Gel Process
}

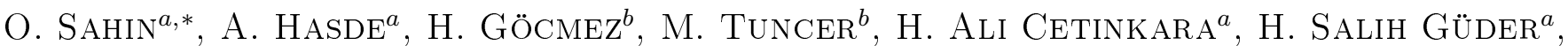 \\ E. SAHIN ${ }^{a}$ AND A. RIZA TUNCDEMIR ${ }^{c}$
}

${ }^{a}$ Department of Physics, Art and Science Faculty, Micro/Nanomechanic Characterization Laboratory Mustafa Kemal University, Hatay, Turkey

${ }^{b}$ Department of Ceramic Engineering, Dumlupinar University, Kutahya, Turkey

${ }^{c}$ Department of Prosthetic Dentistry, Dentistry Faculty, Mustafa Kemal University, Hatay, Turkey

\begin{abstract}
The alumina-zirconia composites are one of the relatively good and promising candidates for biomaterials application, due to biocompatibility and their mechanical properties that combines high flexural strength with a high toughness. The aim of the present work is to analyze the mechanical properties of these composites, where zirconia content was varied from 5 to $50 \mathrm{wt} \%$. The citrate gel method, similar to the polymerized complex method, was used to synthesize these ceramics. Scanning electron microscopy, X-ray diffraction and microhardness tests are used to characterize synthesized materials. The examined material exhibits the behaviour of indentation size effect. Modified proportional resistance model are used to analyze the load dependence of the microhardness. X-ray diffraction analysis was used to calculation of the grain size and dislocation density. It is found that hardness is decreased when the zirconia content increases with enlargement of grain size.
\end{abstract}

DOI: 10.12693/APhysPolA.123.299

PACS: 62.20.-x, 62.20.F-

\section{Introduction}

Zirconia has been used to fabricate dental parts due to their high strength, biocompatibility and fine esthetics. As requests for esthetics increased, selection of zirconia materials has arisen in direct proportion [1] instead of conventional fixed prosthesis. However, it can occur with some problem for bond strength of the veneering porcelain to the zirconia framework. Serious one is cracking of the veneering porcelain and the other is possible degradation of zirconia when exposed to moisture in the oral environment, which may lead to a long term decrease in strength and the possibility of catastrophic failure $[2,3]$. Adding alumina in zirconia or vice versa may decrease these disadvantages $[4,5]$.

Material scientists have been familiar with composites like alumina-zirconia $[6,7]$. Since both materials are biocompatible, this could prove to be a new approach to dental abutments. Although the community of biomaterials has drawn an attention to alumina and zirconia separately, a few studies were published in literature on the use of alumina-zirconia composites as biomaterial [8].

\section{Experimental procedure}

The zirconia-alumina composites were synthesized by the citrate gel method. $\mathrm{ZrOCl}_{2} \cdot 8 \mathrm{H}_{2} \mathrm{O}$ (Fluka, 99\%) and $\mathrm{Al}\left(\mathrm{NO}_{3}\right) \cdot 9 \mathrm{H}_{2} \mathrm{O}$ (Sigma, 98\%) were used as starting materials. Citric acid (Fluka, 99.5\%) and distilled water were used for gelation agent and solvent, respectively. The

*corresponding author; e-mail: osahin@mku.edu.tr experiment was carried out by firstly completely dissolving citric acid into $100 \mathrm{cc}$ of water in a beaker. Then, the required amount of aluminum nitrate and zirconium oxychloride were added respectively into acid solution according to the final compositions containing 5 (A5Z), 15 (A15Z) and 50 (A50Z) vol.\% alumina. The solution was continuously stirred at room temperature until the highly transparent solution was obtained. When the clear solution was obtained, temperature on magnetic stirrer was adjusted to $200^{\circ} \mathrm{C}$ to initiate gelation reaction. As the water evaporated, the viscosity of precursor solution increased and finally turned into a yellowish sticky gel. The gel was conveyed to a mantle heater at $400{ }^{\circ} \mathrm{C}$ for $8 \mathrm{~h}$ to remove organic matter in air.

Finally, the obtained "ceramic gel precursors" was crushed gently in an agate mortar and then repeatedly washed with distilled water, and then suspensions were centrifuged at $9000 \mathrm{rpm}$ for $2 \mathrm{~min}$ and dried in an oven at $100{ }^{\circ} \mathrm{C}$ for overnight. Heat treatment for gel precursors was carried out at $1000^{\circ} \mathrm{C}$ for $2 \mathrm{~h}$. The powders were compacted into disc (with $13 \mathrm{~mm}$ in diameter and $5 \mathrm{~mm}$ in height) at a maximum applied pressure of $200 \mathrm{MPa}$ and subsequently sintered at $1600^{\circ} \mathrm{C}$ for $4 \mathrm{~h}$ in air with a heating and cooling rate of $10^{\circ} \mathrm{C} / \mathrm{min}$.

Hardness measurements were made ranging from at 0.098 to $9.8 \mathrm{~N}$ load with a loading time of $15 \mathrm{~s}$ was used to measure the diagonals of the indentations. The indentation diagonal lengths were measured by Nikon MA 100 inverted metal microscope using Clemex Professional microscopy image analysis software. $50 \times$ and $20 \times$ objective lenses were used on the Nikon MA 100 instrument. In view of the scatter of the microhardness data, the hardness value was a mean of at least six measure- 
ments under the same condition. The hardness values $\left(H_{\mathrm{V}}\right)$ from the length of the two diagonals of the square-shaped Vickers indents were calculated with the equation $H_{\mathrm{K}}=14.229\left(P / d^{2}\right)$ where $P$ is the applied test load in $\mathrm{N}, d$ is the average of two indentation diagonal lengths in $\mu \mathrm{m}$, and 14.229 is a geometrical constant of the diamond pyramid.

\section{Results and discussion}

Figure 1 illustrates X-ray diffraction (XRD) pattern of alumina with addition of various amount of stabilized zirconia. Figure 2 shows microstructure of alumina-zirconia composites. It is stated that grain size and porosity as well as types of phase effect structural properties of ceramic composites. Fine grain size and less porosity in the microstructure are critical issue to get better mechanical properties for such materials.

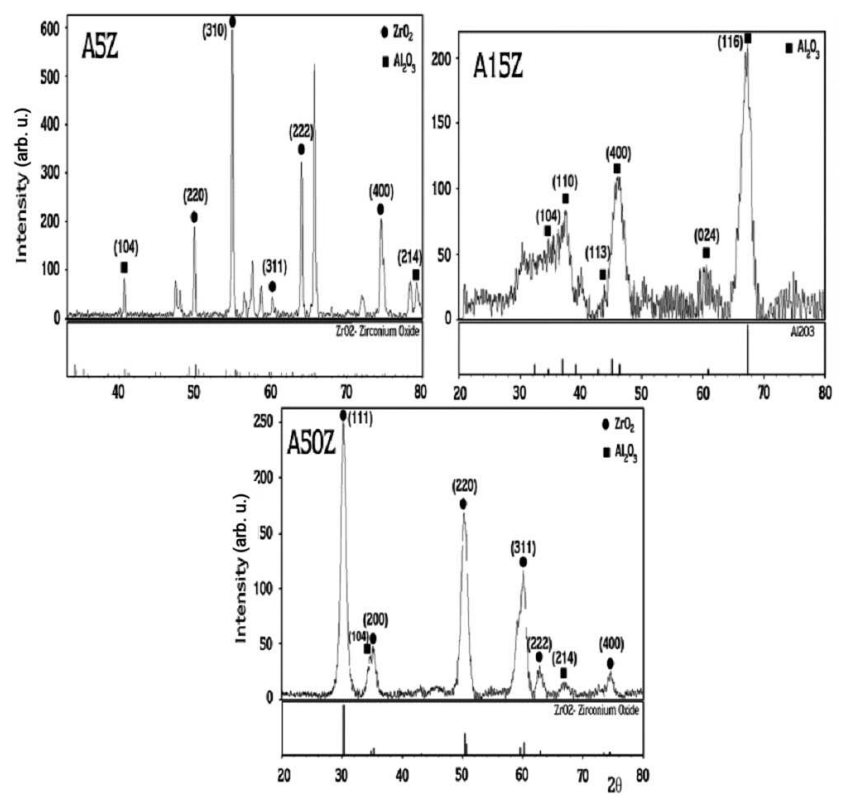

Fig. 1. XRD pattern of A5Z, A15Z, and A50Z ceramics.

Figure 3 shows the variation of $H_{\mathrm{K}}$ as a function of applied test loads ranging from 0.098 to $9.8 \mathrm{~N}$ for all ceramics. The variation of $H_{\mathrm{K}}$ with applied indentation test load for each specimen shows that the $H_{\mathrm{K}}$ decreases with increasing applied indentation test load, and then reaches saturation at about $4.9 \mathrm{~N}$. The entire hardness profile consists of two regimes: load-dependent and load-independent.

Much research work has been performed to explain the origin of the indentation size effect (ISE) and several possible explanations exist. These explanations fall into two sets. The first set, which is the most common explanation found in the literature, concerns the experimental errors resulting from the limitations of the resolution of the objective lens and the sensitivity of the load cell. The

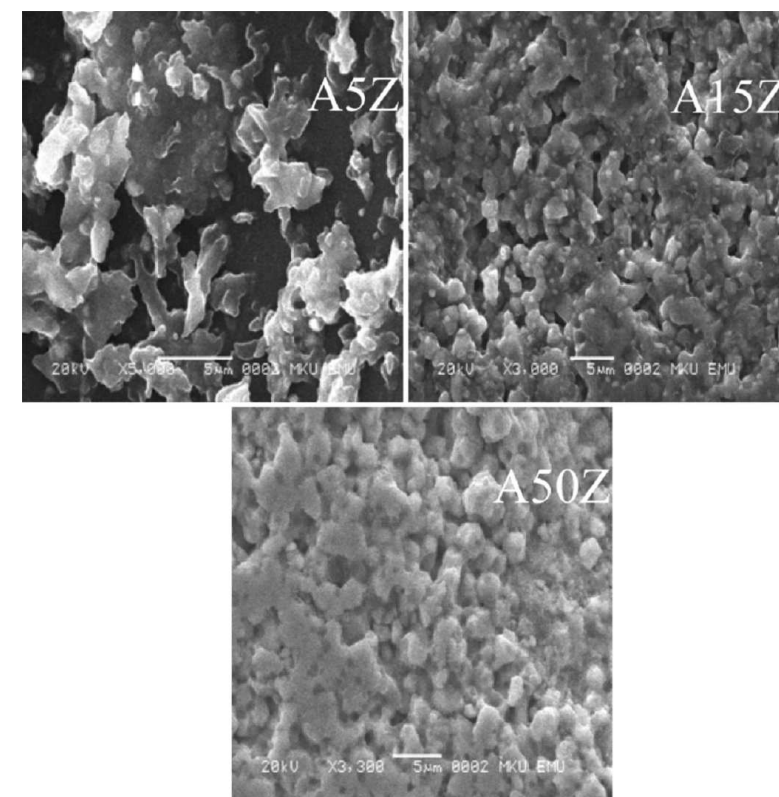

Fig. 2. SEM images of A5Z, A15Z and A50Z.

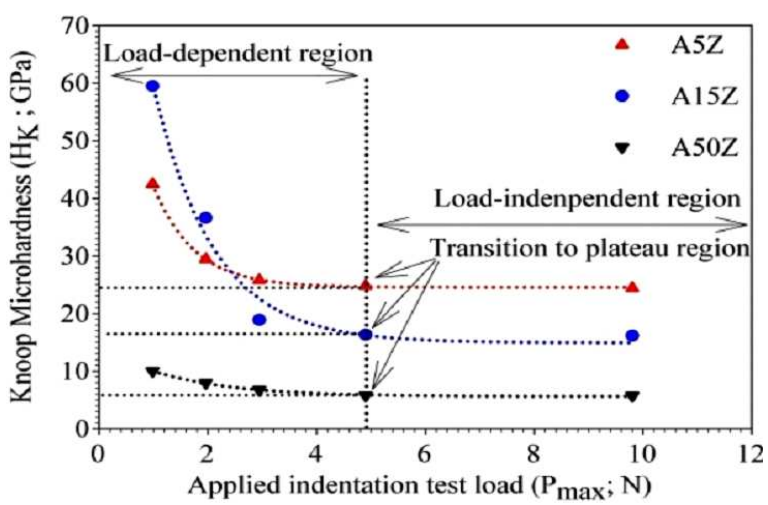

Fig. 3. Knoop microhardness variation as a function of applied indentation test load.

second set is directly related to the intrinsic structural factors of the test materials, including indentation elastic recovery, work hardening during indentation, surface dislocation pining, etc. [9, 10].

The experimental data on dependence of $H_{\mathrm{K}}$ on applied indentation test load can be explained by the HaysKendall approach [11]. They proposed that there exists a minimum applied test load $W$ (test specimen resistance) necessary to initiate plastic deformation, below which only elastic deformation occurs,

$$
P=W+C_{1} d^{2},
$$

where $W$ is the minimum load to initiate plastic deformation and $C_{1}$ is a load-independent constant. From Eq. (1), a plot of $P$ versus $d^{2}$ would yield a straight line, where the parameters of $W$ and $C_{1}$ can easily be calculated from the intersection point and the slope of the curve, respectively. Replacing $P$ in $H_{\mathrm{K}}=14.229 \frac{P}{d^{2}}$ 
equations by $P-W$, one gets an equation to calculate the load-independent hardness as follows:

$$
H_{\mathrm{K}}=14.229 \frac{P-W}{d^{2}} \text {. }
$$

Such plot for the examined ceramics in the present study is shown in Fig. 4. The correlation coefficient, $r^{2}$, is implying that Eq. (2) provides a satisfactory description of the indentation data for the examined materials.

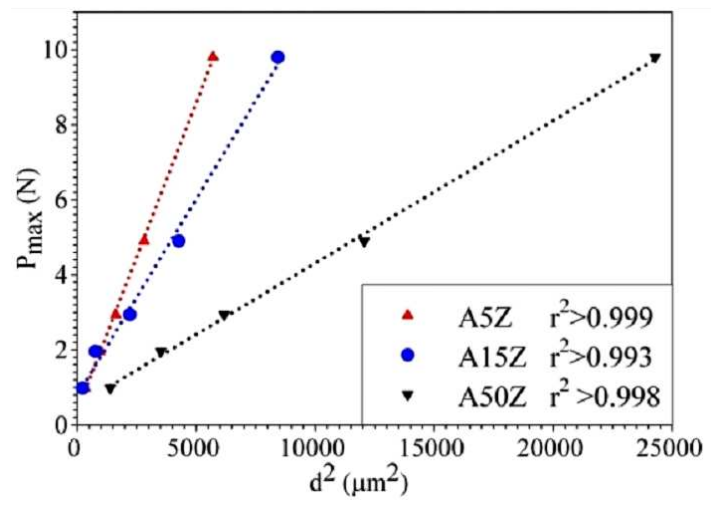

Fig. 4. Plots of applied indentation test load versus indentation test size according to the Hays-Kendall model.

The calculated load-independent microhardness values, $H_{\mathrm{HK}}$, of the ceramics were $23.406,14.897$, and $5.405 \mathrm{GPa}$ for A5Z, A15Z, and A50Z, respectively. These values are very close to the plateau region in Fig. 1.

The crystallite size $(D)$ was calculated using Scherrer's relation [12],

$$
D=\frac{k \lambda}{\beta \cos \theta} .
$$

The microstrain and dislocation density were obtained by using the below relations [12]:

$$
\begin{aligned}
& \varepsilon=\frac{\beta \cos \theta}{4}, \\
& \rho=\frac{15 \varepsilon}{a D},
\end{aligned}
$$

respectively, where $\lambda$ is the wavelength of radiation, $k$ is a constant (0.94), $\beta$ is the full-width at half-maximum, and $\theta$ is the diffraction angle. The crsytallite size, $D$, of the ceramics were 165,187 , and $258 \mathrm{~nm}$ for A5Z, A15Z, and A50Z, respectively. According to the Hall-Petch relation, hardness is inversely proportional to the square root of grain size. As an agreement with Hall-Petch relation, it is observed that the grain refinement has increased the hardness. Hence, high hardness of the A5Z can be attributed to the smaller grain size.
The calculated dislocation density values, $\rho$, of the ceramics were $2.93 \times 10^{16} \mathrm{~cm}^{-2}, 1.98 \times 10^{16} \mathrm{~cm}^{-2}$, and $1.10 \times 10^{16} \mathrm{~cm}^{-2}$ for A5Z, A15Z, and A50Z, respectively [13]. To correlate the variation of hardness with dislocation density of a crystal, there is considered the definition of hardness generally accepted by all. According to this, hardness definition of a material is the resistance to the dislocation motion. Occurrence of this increase may be due to both pinning of dislocations at the impurity sites and other defects caused by the presence of impurity atoms in the crystal, and also to variation in the magnitude of the bond forces in the crystal containing impurities. It was seen that the indentation microhardness values increased with increasing alumina content. It implies that hindering of dislocations motion is critical issue in hardening of the ceramics.

\section{Acknowledgments}

The financial support from research foundation of Mustafa Kemal University (grant No. 09 M 2004 and 08 F 0501) is gratefully acknowledged.

\section{References}

[1] N.V. Raptis, K.X. Michalakis, H. Hirayama, Int. J. Perio. Restor. Dent. 26, 31 (2006).

[2] B. Taskonak, B.A. Borges, J.J. Mecholsky, K.J. Anusavice, B.K. Moore, J. Yan, Dent. Mater. 24, 1149 (2008)

[3] M.V. Swain, Acta Biomater. 5, 1668 (2009)

[4] S. Reich, M. Wichmann, E. Nkenke, P. Proeschel, Eur. J. Oral. Sci. 113, 174 (2005)

[5] W. Att, K. Stamouli, T. Gerds, J.R. Strub, Acta Odontol. Scand. 65, 14 (2007)

[6] D. Casellas, I. Ràfols, L. Llanes, M. Anglada, Int. J. Refract. Met. Hard Mater. 17, 11 (1999)

[7] G. Gregori, W. Burger, V. Sergo, Mater. Sci. Eng. A 271, 401 (1999)

[8] C. Piconi, G. Maccauro, Biomaterials 20, 1 (1999).

[9] O. Sahin, O. Uzun, U. Kolemen, B. Duzgun, N. Ucar, Chin. Phys. Lett. 22, 3137 (2005)

[10] J. Gong, J. Wu, Z. Guan, J. Eur. Ceram. Soc. 19, 2625 (1999)

[11] C. Hays, E.G. Kendall, Metallurgy 6, 275 (1973)

[12] A. Suresh, K. Chatterjee, V. KR. Sharma, S. Ganguly, K. Kargupta, D. Banerjee, J. Electron. Mater. 38, 449 (2009)

[13] A. Hasde, M.Sc. Thesis, Mustafa Kemal University, 2011. 